\title{
Evaluación de la eficiencia en bibliotecas
}

\author{
Nora A. DELgado TO RRES \\ HILDA A. SOSA SAURA \\ CarreradeInformaciónCientífico-TécnicayBibliotecología, \\ Facultad deCo municación, Universidad deLaHabana, LaHabana, Cuba, \\ Tels. 3265 83, 309885 y 323734 Fax (537) 322757
}

\begin{abstract}
RESUMEN
Seexponeunmétodo obtenido como resultadodeinvestigacio nesrealizadas en dos periodos: 1986-1990 y 1994-1995, que se propuso medirlaeficiencia deunabiblio tecaapartirdelosresultadosquedebeal can zarparacum plircon sus objetivos. El propósito fundamental es crear un método por medio del cual lacon duc ción del perso nal seaeficaz yselo greasignarlelatareaadecua daalaperso nain dicadaparaob tenerel resul tado esperado.Seincluyen suge rencias para implantar este método.
\end{abstract}

\begin{abstract}
ABST RACT
A method is described resulting from research carried out in two periods from 1986- 1990 and from 1994-1995, which ai med at gau ging the effi ciency of a library on the basis of the resultsrequired isto ful fill its objectives. The fun damen tal purpo seisto createamethod which when im plemen ted would lead to efficient management of personnel and the right tusk seing assigned to the right person in orderto ob tain the desired results. Sugges tionsareincluded on the implementation of this method.
\end{abstract}

el 15 de agosto de 1997

Trabajo aceptado

el 8 de enero

de 1998
T a gerencia de un sistema de información debe encaminarse, en primera insـtancia, a definir los métodos para la medición de sus resultados, pues un enfoque económico en cualquier actividad sólo es posible cuando se cuenta con métodos y medios para perfeccionar la planificación, organización y evaluación del trabajo y el personal.

En el presente trabajo se expone un método obtenido como resultado de investigaciones realizadas en dos periodos: 1986-1990 y 1994-1995, con el propósito de medir la eficiencia de una biblioteca a partir de los resultados que debe alcanzar y sus costos, para cumplir con los objetivos.

Este trabajo responde al enfoque de evaluación tarea-personal-costos, desde la perspectiva gerencial de los recursos humanos. Es decir, el objetivo fundamental se dirige a crear un método por medio del cual “[...]la conducción del personal 
sea eficaz, y por ende, se logre asignar la tarea adecuada a la persona indicada, para obtener el resultado esperado," 1 con los costos apropiados, toda vez que "[...]correr el riesgo de asignar tareas de alta o mediana complejidad al personal de regular capacidad o que esté en período de entrenamiento o viceversa, puede afectar considerablemente el logro de los objetivos propuestos por la institución[...]"2

Ahora bien, la retroalimentación que proporcionan la asignación adecuada de tareas y la evaluación objetiva del personal puede contribuir en gran medida a la satisfacción laboral, al aumento del desempeño de los empleados y al incremento de su sentido de pertenencia en las unidades de información -aspecto bastante desatendido en la región latinoamericana-, así como al mejoramiento de las relaciones entre subordinados y autoridades, en circunstancias que se caracterizan por la necesidad de cambio y la adaptación a nuevos contenidos y productos.

La aplicación de este método permitirá controlar los recursos humanos y materiales de las unidades de información, con acciones encaminadas hacia los siguientes objetivos:

a) E stablecer los resultados esperados y sus tareas asociadas.

b) Asignar las tareas según la complejidad que requiere su realización.

c) Determinar indicadores e índices de tiempo y costo, para medir tareas y productos.

d) D efinir requisitos de personal.

e) Elaborar programas de adiestramiento y superación.

f) Asignar recursos a la obtención de los resultados definidos.

A estos objetivos generales pueden añadirse otros más específicos, que deben repercutir directamente sobre los trabajadores y que son:

1. Establecer parámetros de valoración que disminuyan el componente subjetivo de la evaluación.

2. Lograr la satisfacción con el trabajo en general y con su cargo en particular.

3. Alcanzar mayores rendimientos laborales y de gestión.

Con los resultados de su aplicación y atendiendo al enfoque de evaluación expuesto, se podrán entonces asignar las tareas determinadas como más complejas, al personal que tenga las mejores condiciones para ejecutarlas.

Asimismo, se podrán establecer las deficiencias técnicas que presente un trabajador, para poder brindarle una atención personalizada que lo haga más útil, además de tener localizados a quienes deben optar por cursos de capacitación y superación acordes con su perfil.

Se afirma que en un sistema de información "[...]para cumplir una tarea, no se debe emplear a una persona que tenga más experiencia o capacidad de la exigida

1 G. A. Gorene. "Planeación estratégica aplicadaalosrecursoshumanos."

2 Pauline Atherton. Manual para sistemasyservicos deinfomaión 
por la tarea a realizar;" 3 del mismo modo "[...]no se debe efectuar ningún trabajo cuyo valor no pueda ser demostrado claramente a las personas que lo realizan" 4 pues se considera que "[...]en una sociedad que se basa en la información, los recursos humanos constituyen el factor esencial." 5

Para implantar este sistema se debe disponer previamente de una escala para emplearla como unidad de medida en la valoración de las diferencias entre las diversas tareas y las características de los empleados de la entidad. La preparación de la escala consiste en:

* Seleccionar y definir los factores comunes a las tareas y al personal que ha de valorarse.

* D eterminar los grados que se deben asignar a cada factor y definirlos.

* Valorar los factores y atribuirles una puntuación por grados.

* Agrupar las tareas con base en la suma de los puntos asignados a cada grado.

O btenerlosvalo resto tales o pun tuación final decadatareayem pleado evalua do.

En tanto se miden y valoran tareas y personas, se determinó hacer un modelo de evaluación para la tarea y otro diferente para la evaluación del personal.

El método contiene los siguientes pasos esenciales:

I. D eterminar los productos finales del área de trabajo y de cada cargo técnico.

II. Listar el total de las distintas tareas que se realizan por producto.

III. D efinir la relación de cada tarea con los productos finales y clasificar las tareas.

IV. O btener los datos primarios.

V. D eterminar la eficiencia por tarea/ producto.

VI. Evaluar tareas y personal.

A continuación se muestran los diferentes procedimientos que integran este método, ejemplificados con algunos resultados.

\section{DETERMINAR PRODUCTOS FINALES}

El concepto de producto final fundamental de un sistema permite definir los resultados que éste debe garantizar para cumplir sus objetivos; y cuando éstos no se han formulado de modo preciso, este concepto facilita la definición de productos y objetivos.

Por ello, conviene utilizar este concepto no sólo como base para definir cuáles son, de entre todos los productos o resultados finales que se obtienen, los que

3 Ibidkm

4 Ibidam

5 Van House, N.A. "Research of theeco no mics oflibraries." LibraryTrends(Illinois) 32 (4) 1984. 
realmente se corresponden con los objetivos, sino también para lograr una mayor claridad en la formulación de los propios objetivos del área que se investiga, como paso preliminar al estudio de las tareas.

Asimismo, al relacionar los contenidos de cada cargo con los productos que se van a obtener, se garantiza la jerarquización de las tareas y su distribución, de acuerdo con los fines y metas del sistema 0 área.

Ejemplos de algunos productos finales:

* Enelpro cesamientoanalítico- sintético delainformació:do cumentosprocesados, vocabularios controlados, bases de datos bibliográficas y factográficas.

* En la selección y adquisición: plan de compras, lista de nuevas adquisiciones, resultados de estudios bibliométricos.

* En el servicio de información: bibliografías, investigaciones temáticas y todo tipo de servicios y publicaciones informativas.

\section{LIST AR TAREAS}

A partir de criterios ofrecidos por jefes y trabajadores de base pueden recopilarse todas las tareas que se ejecutan en el área que se investiga. Es preferible aceptar en principio la denominación que ellos les den y verificar con posterioridad, si fueron exhaustivamente listadas; si no, debe repetirse el proceso hasta tener todas las tareas.

D espués debe nombrarse cada tarea con la terminología técnicamente reconocida, para confeccionar las listas que permitan recoger los datos y establecer comparaciones.

Ejemplos: Tareas del área de procesamiento:

* Procesar los documentos adquiridos: catalogar, clasificar, asignar epígrafes, descriptores o palabras clave, elaborar resúmenes...

* Habilitar documentos para el servicio.

* Confeccionar y actualizar vocabularios controlados.

\section{DETERMINAR RELACIÓN TAREA-PRODUCTO FINAL Y CLASIFICAR LAS TAREAS}

Una vez definidos los objetivos, productos finales y tareas, se procede a determinar el grado de participación de cada una de ellas, en la obtención de algún producto final.

Es conveniente que participen en este paso metodológico, el jefe técnico del área y sus especialistas de mayor experiencia, por dos razones principales: primera, porque resulta complejo lograr una correspondencia exacta entre las tareas y pro- 
ductos finales; y segunda, porque establecer una relación errónea entre ellas viciaría todo el trabajo posterior.

Ya en términos de los pasos sucesivos, esta colaboración tiene además la ventaja de dejar establecidos nexos de trabajo que facilitarán la incorporación del personal del área a la investigación, pues en ocasiones, estas mediciones pueden crear inseguridad en los trabajadores sobre sus implicaciones en cuanto a posibles cambios en sus tareas habituales o en los resultados que se esperan de ellos.

Los trabajadores saben que su rendimiento será evaluado, pero a menudo existen criterios sobre la comprensión de su trabajo y algunos consideran que nadie puede entender cabalmente el proceso, las demandas y las complejidades de su labor. Estas situaciones pueden crear resistencias y actitudes no cooperativas. Por tanto, una comunicación clara puede hacer mucho para minimizar estas reacciones y propiciar un buen clima de trabajo, tanto para la investigación, como para acciones posteriores.

Ejemplo de relación tarea-producto final:

Tarea: Habilitar documentos para el servicio. Producto Final: Documento acuñado, marbeteado y juego de fichas de Autor, Título, Materias.

Cuando queda establecida la correspondencia tareas-productos finales, se procede a la clasificación de las tareas en cuatro categorías, para precisar el carácter y la distribución de las tareas pertenecientes a cada cargo, área o sistema, como sigue:

Tareas básicas: Tareas técnicas que conducen a la obtención del producto final fundamental del área, cargo o sistema. Ejemplo: en el área de procesamiento, catalogar, clasificar, asignar epígrafes.

Tareas espeializadas Tareas de carácter metodológico o de desarrollo que no determinan directamente la obtención del producto final fundamental, pero que influyen en la formación del personal y, por tanto, en su capacidad para ejecutar con mayor calidad las tareas básicas. Ejemplo: la tarea de investigar en cualquier cargo, área o sistema.

Tarms complementanias: Tareas de rutina que complementan las acciones técnicas para la obtención del producto final o tareas relacionadas con productos finales no fundamentales; de acuerdo con el nivel que se analice. Las tareas de rutina pueden ser también básicas de otro nivel o cargo inferior. Ejemplos: para un cargo de especialista en procesos, actualizar el fichero de autoridades; para el área de procesos, calcular el índice de permanencia documentaria; para el sistema, realizar el control de inventario de muebles y equipos.

Tareas nocomespondientes Tareas que deben ser realizadas por otro cargo, área 0 sistema. Ejemplos: para el cargo de especialista en procesos, preparación física de los documentos; para área de servicios, control del plan de adquisiciones; para el sistema, distribución de libros de texto en una biblioteca docente. 


\section{OBTENER DATOS PRIMARIOS}

El cómputo de tiempo dedicado a cada tarea se realiza a partir de un modelo, donde se relacionan todas las tareas que pertenecen al objeto de investigación, y para cada una de ellas, el propio personal que las realiza consigna el tiempo empleado y la cantidad de productos obtenidos diariamente.

El periodo de aplicación del modelo-encuesta no debe exceder de una semana dentro de cada mes, para evitar el agotamiento y posible desinterés, con la consiguiente inexactitud de los datos registrados.

También deben seleccionarse siempre semanas diferentes, es decir, si en el primer mes se escogió la primera semana, en el próximo mes se seleccionaría la segunda, y así sucesivamente. Esto permite la incorporación y análisis de nuevas tareas que pueden surgir, y obtener, a la vez, una muestra representativa del desarrollo del trabajo.

Por otra parte, el modelo recoge las tareas realizadas durante cada día de la semana, ofrece información sobre su cantidad diaria, y permite analizar la organización individual y colectiva de los contenidos de trabajo, pues como se conoce, la diversidad de tareas diarias consume un tiempo improductivo, que atenta irremediablemente contra el aumento de la productividad de trabajo y de su calidad.(Anexo 1)

\section{DETERMINAR LA EFICIENCIA POR TAREA-PRODUCTO}

Al cuantificar el tiempo invertido en cada una de las tareas, el valor numérico obtenido se convierte en un indicador para la planificación, organización y evaluación del trabajo del cargo, área o sistema, en correspondencia con el nivel que se investiga.

Las variables a medir son:

T - cantidad de Trabajadores que realizan cada tarea;

P- Productos obtenidos en cada tarea, sean fundamentales o no;

S- Salario mensual de los trabajadores;

I - tiempo Individual dedicado a cada tarea;

Ie-tiempo de trabajo Individual estipulado (plantilla, contrato, etcétera).

Una vez determinados los valores de las variables, se establecen índices que permiten precisar en qué medida el cargo, área o sistema está capacitado para alcanzar sus objetivos, a partir del logro de los productos finales ya definidos.

Se proponen algunos criterios de evaluación y varios índices de capacidad, tomando como base las magnitudes de las variables declaradas anteriormente.

Para el análisis del tiempo dedicado a las tareas y a la obtención de productos, se realiza el cálculo de los índices siguientes:

Tiempo total mensual del área (real trabajado): (M)

$\mathrm{M}=\sum \mathrm{h}$ sumatoria de las horas trabajadas en todas las tareas del área. 


\section{Tiempo estipulado para el trabajo: (Me)}

$\mathrm{Me}=\mathrm{Ie} \times \mathrm{T}$ : Tiempo total de trabajo mensual estipulado para el área, donde

Ie= Tiempo individual total estipulado de trabajo.

$\mathrm{T}=$ Total de trabajadores del área.

Tiempo dedicado colectivamente a una tarea: (C)

$$
\mathrm{C}=\sum \mathrm{I}
$$

$\sum \mathrm{I}=$ Sumatoria de todos los tiempos individuales dedicados a una tarea

Tiempo máximo posible de dedicación a una tarea: (TM)

$$
\mathrm{TM}=\mathrm{Tt} \times \mathrm{ME}
$$

donde:

$\mathrm{Tt}=(\mathrm{C} \times 100) \div \mathrm{M} \%$ Tiempo real dedicado colectivamente a la tarea.

La obtención del tiempo máximo posible que hay que dedicar a una tarea permite emplear esa cuantificación en la definición de las afectaciones potenciales a las distintas tareas ante cambios en los tiempos de contratación de la mano de obra, las enfermedades o licencias de los trabajadores, su designación para cursos de capacitación, y otras circunstancias, lo que facilita el establecimiento de prioridades y dedicaciones a los productos finales.

\section{Tiempo dedicado a la obtención de cada producto final: (Tp)}

$\mathrm{Tp}=\mathrm{C} \div \mathrm{P}=$ Tiempo dedicado a la obtención de cada Producto.

D espués de realizados los cálculos anteriores, se puede calcular la capacidad de ejecución de producto o tarea en el área dada.

Tanto el índice TM como el Tppermiten estimar los límites del área para asimilar productos o tareas de la índole analizada. Si se vincularan ambos índices se obtendría la Capacidad de Producción o Ejecución

(CP) del área

$$
\mathrm{CP}=[\mathrm{TM} \div \mathrm{Tp}]=[\mathrm{Tt} \times \mathrm{Me}] \div[\mathrm{C} \div \mathrm{P}]
$$

El tiempo global (TM) dedicado a la tarea - digamos catalogar- dividido entre el tiempo promedio (Tp) que demora obtener uno de sus productos -digamos, una ficha catalográfica- indica la cantidad de productos, en este caso fichas, que pueden obtenerse en ese tiempo.

El total de tiempo trabajado realmente en un mes permite estimar de modo directo, la capacidad del área para obtener determinado producto, lo cual puede emplearse, entre otras aplicaciones, para comparar la productividad actual entre centros, y para definir la cantidad de productos que puede asimilar un área dada, si se mantienen similares condiciones de trabajo.

Por otra parte, si se considera que algunas tareas distintas se agrupan para conformar un único producto final, entonces el tiempo total necesario para ese 
producto estaría dado por la sumatoria de los tiempos individuales de las tareas componentes.

Ejemplo:

\begin{tabular}{|l|c|c|c|}
\hline \multicolumn{1}{|c|}{ Tareas } & $\begin{array}{c}\text { Tiempo } \\
\text { dedicado }\end{array}$ & \multicolumn{1}{c|}{$\begin{array}{c}\text { Cantidad } \\
\text { productos }\end{array}$} & $\begin{array}{c}\text { Tiempo } \\
\text { porproducto }\end{array}$ \\
\hline Catalogar & $34 \mathrm{~h}$. & 82 & $24,9 \mathrm{~min}$. \\
\hline Clasif. e indizar & $46 \mathrm{~h}$. & 59 & $46,8 \mathrm{~min}$ \\
\hline Preparac. física & $10 \mathrm{~h}$. & 901 & $6,6 \mathrm{~min}$ \\
\hline $\begin{array}{l}\text { Reproduc. } \\
\text { Fichas }\end{array}$ & $58 \mathrm{~h}$. & 187 & $18,6 \mathrm{~min}$. \\
\hline TOTALES & $148 \mathrm{~h}$. & -- & $96,9 \mathrm{~min}$. \\
\hline
\end{tabular}

La obtención de un libro procesado, como producto final, consume algo más de una hora y media, como promedio, en el periodo analizado. Si se valora el tiempo individual de cada tarea -que fluctúa entre 6 y 49 minutos-, habrá que dedicar distinta cantidad de personal a la realización de cada una, a fin de mantener un ritmo estable de trabajo, o adoptar medidas específicas con la ejecución de las tareas cuyo tiempo se desee abreviar.

De mantenerse las mismas condiciones (en las que se dedica el 53\% del tiempo a procesar libros) puede esperarse que los 4 procesadores del departamento (con 1,628 horas de potencial laboral para procesar) tengan una capacidad de producción de 1,008 libros procesados mensualmente, cifra que podría adoptarse en principio como guía de la planificación del trabajo del área.

\section{Medir Eficiencia}

A partir de considerar la eficiencia como la determinación de los costos con los que se alcanzan ciertos objetivos, se establecen variables que hay que medir para el cálculo de dichos costos.

Lo más provechoso sería poder operar con datos reales sobre el tiempo trabajado y los salarios devengados, pero no siempre los controles proporcionan tales datos en la forma, el tiempo y con la fidelidad con que se necesitan. Entonces, se propone realizar cálculos con datos determinados en teoría, para aquellos que no se encuentren en los modelos-encuesta.

Para el análisis de los costos de las tareas y de la obtención de productos se calculan los siguientes índices:

Salario Hora promedio individual: (shi)

$\mathrm{shi}=\sum \mathrm{S} \div(\mathrm{tm} \cdot \mathrm{T})$

D onde:

$\Sigma \mathrm{S}=$ Sumatoria de salario bruto mensual.

tm= tiempo de trabajo individual estipulado mensual (teórico).

$\mathrm{T}=$ Total de trabajadores del área. 
Esto permite encontrar un índice en el que se eliminen las diferencias salariales que existen entre trabajadores que realizan una misma tarea y facilita el cálculo de costo por actividad.

Costo por actividad (a):

$$
\mathrm{a}=\mathrm{sii} \cdot \mathrm{C}
$$

donde:

shi $=$ salario hora individual.

$\mathrm{C}=$ tiempo colectivo dedicado a la tarea (suma de los tiempos en los modelos-encuesta.)

Costo por producto:

$$
\mathrm{p}=\mathrm{a} \div \mathrm{P}
$$

donde:

$\mathrm{P}=$ suman los productos obtenidos en cada tarea.

Tanto el costo por actividad, como el costo por producto permiten establecer comparaciones entre diferentes periodos para una misma actividad y también pueden tomarse como criterios para la planificación, la organización y el control del trabajo en las condiciones actuales y cuando sea necesario incorporar nuevas tareas o desviar el personal hacia otras funciones.

Para alcanzar mayor precisión en los costos, sería conveniente definir el gasto indirecto que corresponde a cada tarea y añadirlo al costo ya calculado, siempre que se cuente con datos sobre el total de gastos administrativos y se pueda conocer el tiempo de trabajo total de la institución en un año.

La división de esos gastos entre ese tiempo ofrece la proporción de gastos administrativos por hora trabajada y cuando se lo multiplica por el tiempo que consumió una tarea, entonces permite conocer sus gastos indirectos y su costo total.

Ejemplo:

Gastos Indirectos (Gi): Proporción gastos administrativos correspondientes a la tarea.

$$
\mathrm{Gi}=(\mathrm{Ga} \div \mathrm{h} / \mathrm{h}) \cdot \mathrm{C}
$$

donde:

$\mathrm{Ga}=$ Total gastos administrativos .

$\mathrm{h} / \mathrm{h}=$ Total de horas-hombre trabajadas en la institución en un año.

$\mathrm{C}=$ Tiempo dedicado a la tarea.

Costo total de tarea $=\mathrm{ca}+\mathrm{Gi}$ 


\section{VALORACIÓN DE TAREAS Y DE PERSONAL}

Para definir la complejidad de las tareas se diseñó un modelo que contiene 13 factores divididos en dos grupos que responden a:

\section{G rupo 1: Factores $G$ enerales requeridos para realizar la tarea}

1. Preparación profesional: grado de preparación teórico-práctica.

2. Experiencia profesional: años de experiencia laboral.

3. Iniciativa: necesidad de tomar decisiones.

4. Responsabilidad sobre el material y los equipos: valor económico de los materiales y equipos que se emplean, perjuicio posible como consecuencia de errores en su manipulación y empleo.

5. Esfuerzo laboral: intensidad del esfuerzo físico, concentración mental y visual.

6. Condiciones de trabajo: aspectos físicos, materiales y ambientales.

7. Medios de trabajo: necesidad de medios técnicos y materiales.

\section{G rupo 2. Factores Específicos de E jecución requeridos para realizar la tarea}

8. Diversidad de operaciones: cantidad y tipo de operaciones.

9. Diversidad temática: cantidad de temáticas de una o más disciplinas.

10. Diversidad de fuentes de información: variedad de tipos de fuentes de información.

11. Nivel de actualización: actualización del aseguramiento informativo, técnico y profesional.

12. Nivel de especialización: el técnico profesional.

13. Periodo de ejecución: tiempo estipulado para concluir la tarea.

Cada uno de estos factores se divide, a su vez, en 5 grados que se definen asignándoles puntos de acuerdo con una escala descendente, donde el primer grado es el superior y el quinto es el inferior (ver Tablas de la 1 a la 13 en anexo).

En este caso, las definiciones de cada grado responden a las condiciones y situaciones propias del país, y a las experiencias recogidas en las entrevistas realizadas. Lógicamente, para su perfeccionamiento y validación se requiere de una prueba piloto que no fue posible realizar por cuestiones de tiempo, lo cual constituye una limitación para su implantación.

Por otro lado, se confeccionan tablas auxiliares donde se encuentra el total de puntos por factor (Tabla 14), la cual ayudará a elaborar la tabla de clasificación de las tareas por puntos (Tabla 15).

Si se tienen estas tablas completas, se puede proceder a evaluar cada una de las tareas escogidas, utilizando la observación o la entrevista directa con quienes la realizan, y llenando la hoja de valoración (Tabla 16), en la que se reflejarán los grados y consiguientes puntos adjudicados a la tarea que se evalúa. La 
suma total obtenida se busca en la Tabla 15, en la que se ubica su clase 0 nivel de complejidad.

Luego de evaluadas todas las tareas técnicas necesarias y ubicadas en su nivel de complejidad, se elabora la tabla de clasificación de las tareas (Tabla 17), que permite tenerlas objetivamente clasificadas y poder cumplimentar los objetivos que se describieron con anterioridad.

En cuanto a la evaluación del personal, el modelo consta de 18 factores, cada uno con su definición más amplia y general, divididos a su vez en dos grupos y son:

\section{Grupo 1: C apacidad y rendimiento de trabajo}

1. Grado de preparación profesional: preparación teórica y práctica del trabajador. Considera la preparación académica u otra pertinente para desempeñar el cargo, así como las habilidades y experiencias obtenidas en el desempeño de la(s) tarea(s).

2. Capacidad de comunicación: habilidad para trasmitir de forma oral o escrita información pertinente, clara, precisa, con lo que se logra captar la atención del receptor y se obtiene una retroalimentación.

3. Rendimiento habitual: capacidad y habilidad para realizar los trabajos asignados en el tiempo previsto y conforme a las normas establecidas de ejecución.

4. Calidad del trabajo: habilidades y destrezas desarrolladas por el individuo para ejecutar la tarea de forma correcta, sin errores ni omisiones, sobre la base de lo estipulado por la institución, para obtener resultados por encima de lo esperado.

5. Rapidez en la ejecución: habilidad del individuo para realizar el trabajo en el tiempo estipulado por la institución, la cual puede ser superada o no alcanzada.

6. Iniciativa: capacidad de proponer y emprender acciones útiles o innovadoras para la agilización y mejor desarrollo del trabajo colectivo o individual.

7. Espíritu de colaboración: capacidad de integración del individuo al trabajo en colectivo y de cooperar en el desarrollo y realización de tareas afines a su trabajo.

8. Interés: preocupación y esfuerzo del individuo por obtener mejores resultados.

9. D ominio de idiomas extranjeros: cantidad y calidad de idiomas que lee, escribe y habla el trabajador.

10. Actitud ante la superación profesional: disposición que demuestra el individuo hacia una proposición de ese tipo.

11. D ominio de nuevas tecnologías: habilidad del individuo para trabajar con los instrumentos tecnológicos requeridos para realizar el trabajo. 


\section{G rupo 2: D isciplina}

12. Comportamiento en el trabajo: actitudes personales y profesionales para con la institución, sus empleados y jefes.

13. Puntualidad.

14. Constancia ante el trabajo: sistematización demostrada tanto en el desempeño de la tarea como su asistencia a él.

\section{Grupo 3: A ptitudes directivas (sólo para empleados con mando o cargos de dirección)}

15. Capacidad de organización: capacidad del individuo para relacionar, integrar y ordenar metódicamente las actividades del personal, equipos de trabajo y otras unidades de servicio con el propósito de alcanzar los objetivos propuestos por la institución que dirige.

16. Respecto a los subordinados: capacidad de relación con los subordinados, demostrada por su actitud de escuchar y asimilar criterios.

17. A tención a la formación de los subordinados: preocupación por la superación de los empleados para determinar proyectos, programas, cursos o entrenamientos para la elevación de su capacitación.

18. Capacidad para asimilar nuevos métodos: actitud ante cambios de cualquier índole en su puesto o unidad de trabajo.

Estos factores se presentan en una hoja Estándar (Tabla 18) que tiene un cuadro con cada factor dividido en 5 grados, cada uno de los cuales tiene asignados puntos en escala ascendente: Óptimo (1); Bueno (2); Medio (3); Escaso (4); Insuficiente (5). Además, estos grados están definidos subsecuentemente para que, a partir de la definición expuesta de cada uno de los factores, se determine qué puntuación asignar al empleado que se evalúa.

El modelo posee una columna final en cada grupo de factores en la que se ubica el puntaje total de cada grado. La última fila del modelo muestra las escalas de puntuación total, tanto para subordinados como para los jefes.

Por otro lado, se confeccionan tablas auxiliares de puntuación por cada uno de los grupos de factores (Tablas 19, 20 y21), las cuales servirán para determinar la escala numérica que el empleado obtiene para asignar la valoración global.

El evaluador podrá ejecutar su función realizando los siguientes pasos:

a) O bservar de modo sistemático el desempeño laboral del trabajador y realizar entrevistas que le permitan determinar qué grado le corresponde al evaluado y asignarlo en la columna de puntuación de la Tabla 18.

b) Luego de asignar los puntos a todos los factores (para los empleados se considera el primer y segundo grupos y para los directivos, todos), debe sumarse cada grupo, y ubicarse en la fila correspondiente a la valoración global. Con estos resultados se remite a las tablas auxiliares de puntuación, donde se ubicará el 
valor global de cada grupo de factores y se colocará al lado de la suma total anterior.

c) Sumar los valores correspondientes a cada una de las valoraciones globales (en el caso de los jefes serían los tres valores globales, para los empleados, sólo dos).

d) Buscar el resultado en la última fila donde aparece la valoración total del empleado (las cifras correspondientes al directivo se encuentran entre paréntesis) y donde cada grupo de números corresponde a la clasificación de óptimo, bueno, medio, escaso, insuficiente. El término con el que se determina la evaluación se coloca en la esquina derecha inferior del modelo.

\section{SU GEREN CIAS PARA IMPLANTAR ESTE MÉTODO}

Se está consciente de que proponer un nuevo modelo de evaluación para aplicar en bibliotecas implica decisiones y disposiciones que van más allá de los límites que tiene este trabajo.

Pueden existir obstáculos legislativos - o gremiales- relativos, por ejemplo, al otorgamiento de puestos, a la contratación de personal, a la asignación de salarios y a las definiciones de tiempos de trabajo. La metodología propuesta para integrar la determinación de tareas, productos, eficiencia y evaluación, no sólo plantea entonces nuevos factores, sino una nueva concepción.

Pero aun si se salvaran esas limitaciones, tendrían que enfrentarse otras más difíciles de vencer, como son las de orden subjetivo o las referidas a mecanismos burocráticos.

No obstante, se considera que proponer este modelo es importante para darle mejor sentido de valoración, no sólo al trabajador actual y a sus contenidos, sino al profesional de la información en las bibliotecas.

Por ello, aunque las condiciones para su aplicación necesitan de la gerencia de recursos humanos y de información planteadas en este trabajo, es posible que cualquier biblioteca aplique de modo experimental este esquema de evaluación para corroborar su efectividad y perfilar su propia proyección, tomando en cuenta el plan de introducción que consiste en:

a) Tener directrices claras en los aspectos de organización de la institución. En este caso, determinar qué departamento asumirá el experimento y con qué personal.

b) Crear, estudiar y aprobar un programa de valoración de tareas y personal por parte de la dirección y los investigadores comprometidos con llevar adelante el estudio.

c) Adiestrar a las personas designadas para realizar el análisis y la valoración de las tareas. Es importante que los evaluadores precisen muy bien cuáles son las funciones de cada tarea que ha de evaluarse, así como la mejor comprensión del 
método; por medio de seminarios se pueden explicar los objetivos, instauración y resultados esperados, para garantizar que exista el mínimo de errores en su aplicación.

d) Aplicación del plan: analizar, describir, valorar y clasificar las tareas que se ejecutan en la biblioteca.

e) Utilización de los resultados de la valoración en los rangos que puedan abarcarse: tareas, productos, personal o áreas de organización, conforme a los resultados obtenidos.

\section{OTRAS CONSIDERACIONES}

No obstante la presentación de este modelo de evaluación con fines de eficiencia y efectividad, es necesario delimitar su función para con las personas, como seres humanos. En este modelo se recogen aspectos que pueden ser satisfechos o no por el evaluado y que al mismo tiempo se les puede apreciar o cuantificar. Pero es necesario aclarar que existen otras cualidades que de ninguna forma pueden incluirse en modelos, aunque sean muy importantes, como aquellas actitudes y manifestaciones relacionadas con la ética de un profesional de la información, no siempre formalizadas en un código.

Por ello, se hará referencia al código de ética elaborado por la Corporación de Bibliotecarios de Québec, ${ }^{6}$ cuyos valores pueden considerarse universales. En esta fuente se detallan cuatro capítulos que tratan aspectos desde la definición de lo que es un bibliotecario, hasta el establecimiento de sus deberes y obligaciones con la sociedad.

Se enfatiza en su obligación de dar accesibilidad a la cultura y a la información sin ninguna discriminación. De igual modo exhorta al bibliotecario para que apoye toda medida susceptible de asegurar servicios profesionales de calidad a la población.

En cuanto a los deberes y obligaciones con el usuario se destacan la integridad, entendida como la lealtad al usuario; honradez en el ejercicio de su profesión, manifestada en el hecho de no sobrepasar su competencia o proporcionar informaciones incompletas, anticuadas, no verificables o inexactas.

Si fuera necesario expresar opiniones, el bibliotecario debe profundizar en el campo del conocimiento relativo al problema. Y de la misma forma, prevenir a su usuario de cualquier error perjudicial que se haya cometido al prestarle el servicio.

La responsabilidad se plantea en el sentido más íntimo del concepto, o sea, garantizar con su trabajo personal cuanto informe o documento brinde, siempre y cuando éste no haya sido modificado sin su conocimiento. Asimismo, el

6 W.F.Lancaster. "Avaliançao deserviçosdebiblio tecas.” F.W.Lan caster.Brasília,D :F.: Bri quet de Lemos/ Livros, 1996. 356 pp. 
bibliotecario hace referencia al secreto profesional, la independencia y la actuación desinteresada.

Estos conceptos parecen obvios; sin embargo, no siempre se consideran expresamente en la valoración del papel del trabajador de la información. Se trata de aspectos cualitativos cuya adopción depende en última instancia de la voluntad personal, pero que tienen una influencia decisiva en el prestigio e imagen de la profesión.

\section{BIBLIOGRAFíA}

Arana, L.; C. Curbelo. Psicooǵa dd trabaja sdeeción dd pesana/ L. Arana y C. Curbelo. La Habana: Dirección de Publicaciones de la UH, 1966.

Arias G alicia, Fernando. Administracón de rearsos humanos / Fernando Arias Galicia. México: Editorial Trillas, 1975.

Atherton, Pauline. Manual para sistemas y servicios deinformaión/ Pauline A therton. París: Unesco, 1978. 343 pp.

Barquero Corrales, A. La administracón dereeursos humanos (1 parte). / A. Barquero Corrales. San José, C.R.: EUNED, 1992.

Calleja, Nazira. "El desempeño y la satisfacción laboral de bibliotecarios: Construcción de escalas de evaluación." Invetigaaón Biblictedóóġa(México) 2(4): 11-24; enero-junio 1988. ISSN 0187-358X

Elorduy Mota, Juan Ignacio. Estrategia dela empresa y reursoshumanos Una visón dnámi ca dela empresa / Juan Ignacio Elorduy Mota. Madrid: McG raw-Hill, 1993. 184 pp. ISBN 84-481-0090.

Gorene, G. A. Planeaión estratégica aplicada a los reursos humanos/ G A. G orene. Bogotá: NORMA, 1985.

Gutzmann, Horst. BuhuundBiblidhdk. LISA (EUA)31 (4), april, 79. pp. 348-354.

Heneman, Herbert. Administracón de reursos humanos y pessonal. / Herbert Heneman. México: CECSA, 1987.

Klingner, D . y Law. L. Descipaón de tareas, nuevos usos como instrumento familiar. LISA (EUA) 72(1), Winter 1979. pp. 65-67.

Lancaster, F. W. "Analysis of cost and performance." Library trends (Illinois) 21(4) 1973.

- -. "Avaliaçao de serviços de bibliotecas." F.W. Lancaster. Brasília, DF: Briquet de Lemos/ Livros, 1996. 356 pp. 
Muñoz Betemps, José Luis. "La función de organización y recursos humanos en procesos de reconversión." Alta Dirección 30(179): 31-36; enero-febrero 1995. ISSN 0002-6549.

Reyes Ponce, A. El análisis depurstos / A. Reyes Ponce. México: Editorial Limusa, 1987.

Riccardi, R. y L. Perego. La valoracón dd pessonal. / R. Riccardi y L. Perego. Barcelona: Editorial Hispano Europea, 1959. 203 pp.

Rodrigo Garrido, José Luis. "Una experiencia de gestión de recursos humanos en un proceso de fusión." Alta Direción (Barcelona) 30(179): 49-54; enero-febrero 1995. ISSN 00026549.

Rodríguez P., J.M. El factor humamo en la empeea/ J.M. Rodrí guez P. Bilbao: D EUSTO, 1992.

Rodríguez, R., Víctor M. Administracón depesanal. / Víctor M. Rodríguez R. Santo D omingo: D.N., 1979.

Tilson, Yvette. "Income generation and pricing in libraries." Library Management (West Yorkshire) 15(2): 5-17; February 1994. ISSN 0143-5124.

Van House, N.A. "Research of the economics of libraries." LibraryTrends (Illinois) 32 (4) 1984.

Vázquez Valero, M. "Suministro de documentos: Costes y precios." Reista Española de Doumentacón Científica. (Madrid) 18(1): 33-41; enero-marzo 1995. ISSN 0210-0614.

Wingand, Darlene E. "What do products/ services cost? How do we know?" LibraryTrends (Illinois) 43(3): 401-408; Winter 1995. ISSN 0024-2594.

Werther, William B. Jr y K. D avis. Administracón depersonal yre arsos humanos William B. Werther Jr y K. Davis. México: McG raw Hill, 1991.

Williams. R. V. "Mediciones de la productividad en las bibliote cas especializadas: perspectivas y problemas." Speial Libraries (EUA) 2(7) Primavera, 1988. 


\section{ANEXO}

\begin{tabular}{|c|c|c|c|c|c|c|c|c|c|c|c|c|c|c|c|c|}
\hline $\mathrm{MO}$ & ELC & & & & & & $\mathrm{m}$ & & & & & & & & & \\
\hline & EAS & & un & & & art & & & $\mathrm{rcc}$ & & & leve & & & em & \\
\hline 11 & 1111 & I & $\mathrm{F}$ & $P$ & $\mathrm{I}$ & $\mathrm{F}$ & $P$ & I & $F$ & $P$ & $\mathrm{I}$ & $F$ & $P$ & $\mathrm{I}$ & $F$ & $P$ \\
\hline 1 & & & & & & & & & & & & & & & & \\
\hline 2 & & & & & & & & & & & & & & & & \\
\hline 3 & & & & & & & & & & & & & & & & \\
\hline 4 & & & & & & & & & & & & & & & & \\
\hline & & & & & & & & & & & & & & & & \\
\hline & & & & & & & & & & & & & & & & \\
\hline & & & & & & & & & & & & & & & & \\
\hline & & & & & & & & & & & & & & & & \\
\hline
\end{tabular}

I = Hora en que inició la tarea.

$\mathrm{F}=$ Hora en que finalizó la tarea.

$\mathrm{P}=$ Cantidad de productos obtenidos.

T ablas para la evaluación de tareas en la biblioteca

Tabla1

\begin{tabular}{|c|l|c|}
\hline \multicolumn{3}{|c|}{ FACTOR 1: PREPARACIÓN PROFESIONAL } \\
\hline GRADO & \multicolumn{1}{|c|}{ DEFINICIÓN } & PUNTOS \\
\hline 10. & Requiere ser realizada por un qraduado universitario de ICT-B & 5 \\
\hline 20. & Puede realizarla un qraduado universitario de otra carrera afín & 4 \\
\hline 30. & $\begin{array}{l}\text { Requiere ser realizada por un graduado de técnico medio en } \\
\text { ICT-B }\end{array}$ & 3 \\
\hline 40. & $\begin{array}{l}\text { Puede realizarla un graduado en técnico medio de otra carrera } \\
\text { af́́n }\end{array}$ & 2 \\
\hline 50. & $\begin{array}{l}\text { Puede realizarla un graduado de Pre-universitario u otro con } \\
\text { conocimientos equivalentes }\end{array}$ & 1 \\
\hline
\end{tabular}


74 Investigacón Biblictedógica v. 12 No. 24 enero/ junio de1998

Tada2

\section{FACTOR2:EX PE RIEN CIAPRO FE SIO NAL}

\begin{tabular}{|c|l|c|}
\hline GRADO & \multicolumn{1}{|c|}{ DEFIN ICIÓN } & PUNTOS \\
\hline 10. & Requiere experiencia mavor de 10 años & 5 \\
\hline 20. & Requiere experiencia de 5 a 10 años & 4 \\
\hline 30. & Requiere experiencia de 1 a 5 años & 3 \\
\hline 40. & Requiere experiencia menor de 1 año & 2 \\
\hline 50. & No requiere ninguna experiencia & 1 \\
\hline
\end{tabular}

Tabla3

\begin{tabular}{|c|l|c|}
\hline \multicolumn{2}{|c|}{ FACTOR 3: INICIATIVA } \\
\hline GRADO & \multicolumn{1}{|c|}{ DEFINICIÓN } & PUNTOS \\
\hline 10. & Completamente independiente, confiada a la iniciativa propia & 5 \\
\hline 20. & $\begin{array}{l}\text { Dificultosa y complicada. Requiere tomar con frecuencia } \\
\text { importantes decisiones v acciones rápidas }\end{array}$ & 4 \\
\hline 30. & $\begin{array}{l}\text { Requiere ocasionalmente decisiones o acciones de cierta } \\
\text { importancia }\end{array}$ & 3 \\
\hline 40. & $\begin{array}{l}\text { Requiere estricta observación de instrucciones y la adopción de } \\
\text { decisiones simples }\end{array}$ & 2 \\
\hline 50. & No tiene ninguna oportunidad de tomar decisiones propias & 1 \\
\hline
\end{tabular}

Tabla4

\begin{tabular}{|c|l|c|}
\hline \multicolumn{3}{|c|}{ FACTOR 4: RESPON SABILIDAD SOBRE EL MATE RIALY EQUI POS } \\
\hline GRADO & \multicolumn{1}{|c|}{ DEFINICIÓN } & PUNTOS \\
\hline 10. & $\begin{array}{l}\text { Se requiere sumo cuidado. Cualquier fallo acarrearía daños } \\
\text { considerables }\end{array}$ & 5 \\
\hline 20. & $\begin{array}{l}\text { Presupone un sentido de responsabilidad superior. Posibilidad de } \\
\text { daños de importancia }\end{array}$ & 4 \\
\hline 30. & $\begin{array}{l}\text { Necesita cierta precaución para evitar daños de mediana } \\
\text { importancia }\end{array}$ & 3 \\
\hline 40. & Requiere mínimo de atención y cautela para evitar daños & 2 \\
\hline 50. & No se pueden presentar daños a los equipos y materiales & 1 \\
\hline
\end{tabular}

\section{Segundo grupo de factores}

Tabla5

\begin{tabular}{|c|l|c|}
\hline \multicolumn{3}{|c|}{ FAC TOR 5:ESFUERZO LA BO RAL } \\
\hline GRADO & \multicolumn{1}{|c|}{ DEFINICIÓN } & PUNTOS \\
\hline 10. & $\begin{array}{l}\text { Requiere elevado esfuerzo mental y visual. Insignificante esfuerzo } \\
\text { físico }\end{array}$ & 5 \\
\hline 20. & Mucho esfuerzo mental v visual. Alqún esfuerzo físico & 4 \\
\hline 30. & Esfuerzo mental, visual v físico promedio & 3 \\
\hline 40. & Poco esfuerzo mental v visual y mucho esfuerzo físico & 2 \\
\hline 50. & Casi no requiere esfuerzo mental y visual. Sí esfuerzo físico & 1 \\
\hline
\end{tabular}


Tabla6

\begin{tabular}{|c|l|c|}
\hline \multicolumn{3}{|c|}{ FACTOR 6: CONDICIONES DE TRABAJO } \\
\hline GRADO & \multicolumn{1}{|c|}{ DEFINICIÓN } & PUNTOS \\
\hline 10. & $\begin{array}{l}\text { Requiere acondicionamiento de local, iluminación central y } \\
\text { especializada, condiciones hiciénicas especiales }\end{array}$ & 5 \\
\hline 20. & $\begin{array}{l}\text { Acondicionamiento de local, iluminación central y buenas } \\
\text { condiciones hiciénicas }\end{array}$ & 4 \\
\hline 30. & Buena ventilación e iluminación central. Condiciones hiciénicas & 3 \\
\hline 40. & Condiciones normales de iluminación y ventilación e hiciene & 2 \\
\hline 50. & No tiene exigencias específicas en las condiciones de trabajo & 1 \\
\hline
\end{tabular}

Tabla7

\begin{tabular}{|c|l|c|}
\hline \multicolumn{3}{|c|}{ FACTOR 7: MEDIOS DE TRABAJO } \\
\hline GRADO & \multicolumn{1}{|c|}{ DEFINICIÓN } & PUNTOS \\
\hline 10. & $\begin{array}{l}\text { Requiere utilización de equipos automáticos y mecánicos } \\
\text { sofisticados }\end{array}$ & 5 \\
\hline 20. & Utilización de equipos automáticos v mecánicos de cualquier índole & 4 \\
\hline 30. & En ocasiones debe utilizar equipos automáticos v mecánicos & 3 \\
\hline 40. & En muv pocas ocasiones utiliza equipos automáticos o mecánicos & 2 \\
\hline 50. & No requiere equipos de ningún tipo, es totalmente manual & 1 \\
\hline
\end{tabular}

\section{Tencer grupo de factores}

Tabla8

\begin{tabular}{|c|l|c|}
\hline \multicolumn{3}{|c|}{ FACTOR 8: DIVERSIDAD DE OPE RACIONES } \\
\hline GRADO & \multicolumn{1}{|c|}{ DEFIN ICIÓN } & PUNTOS \\
\hline 10. & Realiza indispensablemente una amplia diversidad de operaciones & 5 \\
\hline 20. & $\begin{array}{l}\text { En muchas ocasiones realiza una considerable diversidad de } \\
\text { operaciones }\end{array}$ & 4 \\
\hline 30. & En alqunas ocasiones requiere mucha diversidad de operaciones & 3 \\
\hline 40. & Tiene poca diversidad de operaciones & 2 \\
\hline 50. & No tiene diversidad de operaciones. Es monótono & 1 \\
\hline
\end{tabular}

Tada 9

\begin{tabular}{|c|l|c|}
\hline \multicolumn{3}{|c|}{ FACTOR 2: EXPERIENCIA PROFESIONAL } \\
\hline GRADO & \multicolumn{1}{|c|}{ DEFINICIÓN } & PUNTOS \\
\hline 10. & Requiere experiencia > 10 años & 5 \\
\hline 20. & Requiere experiencia de 5 a 10 años & 4 \\
\hline 30. & Requiere experiencia de 1 a 5 años & 3 \\
\hline 40. & Requiere experiencia < 1 año & 2 \\
\hline 50. & No requiere ninguna experiencia & 1 \\
\hline
\end{tabular}


76 Investigadón Biblideedógica V. 12 No. 24 enero/ junio de1998

Tabla 10

\begin{tabular}{|c|l|c|}
\hline \multicolumn{3}{|c|}{ FACTOR 3: INICIATIVA } \\
\hline GRADO & \multicolumn{1}{|c|}{ DEFINICIÓN } & PUNTOS \\
\hline 10. & Completamente independiente, confiada a la iniciativa propia & 5 \\
\hline 20. & $\begin{array}{l}\text { Dificultosa y complicada. Requiere tomar con frecuencia } \\
\text { importantes decisiones v acciones rápidas }\end{array}$ & 4 \\
\hline 30. & Requiere ocasionalmente decisiones o acciones de cierta importancia & 3 \\
\hline 40. & $\begin{array}{l}\text { Requiere estricta observación de instrucciones y la adopción de } \\
\text { decisiones simples }\end{array}$ & 2 \\
\hline 50. & No requiere tomar decisiones propias & 1 \\
\hline
\end{tabular}

Tabla 11

\begin{tabular}{|c|l|c|}
\hline \multicolumn{3}{|c|}{ FACTOR 11: NIVELDE ACTUALIZACIÓN } \\
\hline GRADO & \multicolumn{1}{|c|}{ DEFINICIÓN } & PUNTOS \\
\hline 10. & Toda la información utilizada requiere de alto nivel de actualización & 5 \\
\hline 20. & $\begin{array}{l}\text { La mayor parte de la información utilizada requiere alto nivel de } \\
\text { actualización }\end{array}$ & 4 \\
\hline 30. & En ocasiones requiere información de alto nivel de actualización & 3 \\
\hline 40. & Casi nunca exiqe información de alto nivel de actualización & 2 \\
\hline 50. & No requiere información de alto nivel de actualización & 1 \\
\hline
\end{tabular}

Tabla 12

\begin{tabular}{|c|l|c|}
\hline \multicolumn{3}{|c|}{ FACTOR 12: NIVELDE ESPECIALIZACIÓN } \\
\hline GRADO & \multicolumn{1}{|c|}{ DEFINICIÓN } & PUNTOS \\
\hline 10. & Indispensable utilización de información altamente especializada & 5 \\
\hline 20. & Casi siempre necesita información altamente especializada & 4 \\
\hline 30. & En ocasiones utiliza información altamente especializada & 3 \\
\hline 40. & Casi nunca se utiliza información altamente especializada & 2 \\
\hline 50. & No requiere información altamente especializada & 1 \\
\hline
\end{tabular}

Tabla 13

\begin{tabular}{|c|l|c|}
\hline \multicolumn{3}{|c|}{ FACTOR 13: PERIODO DE EJECUCIÓN } \\
\hline GRADO & \multicolumn{1}{|c|}{ DEFINICIÓN } & PUNTOS \\
\hline 10. & Muv urgente, tiempo muy corto de ejecución y culminación & 5 \\
\hline 20. & Menos urgente, corto periodo de ejecución y culminación & 4 \\
\hline 30. & Tiempo normal estipulado para su ejecución y culminación & 3 \\
\hline 40. & Prolongado tiempo relativo para su ejecución y culminación & 2 \\
\hline 50. & No tiene exigencias en cuanto al periodo de ejecución y culminación & 1 \\
\hline
\end{tabular}


Tabla 14

\begin{tabular}{|c|c|c|c|c|c|}
\hline \multicolumn{7}{|c|}{ Puntuación por factores } \\
\hline GradoFactor & $\mathbf{1 0 .}$ & $\mathbf{2 0 .}$ & $\mathbf{3 0 .}$ & $\mathbf{4 0 .}$ & $\mathbf{5 0 .}$ \\
\hline 1 & 5 & 4 & 3 & 2 & 1 \\
\hline 2 & 5 & 4 & 3 & 2 & 1 \\
\hline 3 & 5 & 4 & 3 & 2 & 1 \\
\hline 4 & 5 & 4 & 3 & 2 & 1 \\
\hline 5 & 5 & 4 & 3 & 2 & 1 \\
\hline 6 & 5 & 4 & 3 & 2 & 1 \\
\hline 7 & 5 & 4 & 3 & 2 & 1 \\
\hline 8 & 5 & 4 & 3 & 2 & 1 \\
\hline 9 & 5 & 4 & 3 & 2 & 1 \\
\hline 10 & 5 & 4 & 3 & 2 & 1 \\
\hline 11 & 5 & 4 & 3 & 2 & 1 \\
\hline 12 & 5 & 4 & 3 & 2 & 1 \\
\hline 13 & 5 & 4 & 3 & 2 & 1 \\
\hline T0TAL & $\mathbf{6 5}$ & $\mathbf{5 2}$ & $\mathbf{3 9}$ & $\mathbf{2 6}$ & $\mathbf{1 3}$ \\
\hline
\end{tabular}

Tabla 15

\begin{tabular}{|l|l|}
\hline \multicolumn{1}{|c|}{ SUMA DE PUNTOS } & NIVELES DE COMPLEJIDAD \\
\hline DE 53 A 65 & PRIMERO \\
\hline DE 43 A 52 & SEGUNDO \\
\hline DE 33 A 42 & TERCERO \\
\hline DE 23 A 32 & CUARTO \\
\hline DE 13 A 22 & QUINTO \\
\hline $\begin{array}{l}\text { NOTA: Aunque el rango de movimiento es 10,6, como nunca se } \\
\text { otorgan evaluaciones fraccionarias, se tomó 10 como rango para } \\
\text { establecer las clases y se ajus tó ladiferen ciaglo bal de3 pun tos, en el } \\
\text { mayor niveldecomplejidad,difícilmentealcanzable. }\end{array}$ \\
\hline
\end{tabular}


78 Investigaaón Biblideedógica v. 12 No. 24 enero/ junio de 1998

Tabla 16

\begin{tabular}{|c|c|c|c|c|c|c|c|}
\hline & Características & Óptimo(1) & Bueno(2) & Medio(3) & Escaso(4) & Insuficiente(5) & Pto \\
\hline 1 & $\begin{array}{l}\text { Preparación } \\
\text { profesional. }\end{array}$ & Excelente & Notable & Normal & Mediano & Nulo & \\
\hline 2 & $\begin{array}{l}\text { Capacidad } \\
\text { comunicación. }\end{array}$ & Excelente & Bueno & Normal & Poca & Ninguna & \\
\hline 3 & $\begin{array}{l}\text { Rendimiento } \\
\text { habitual. }\end{array}$ & Elevado & Bueno & Suficiente & Escaso & Insuficiente & \\
\hline 4 & $\begin{array}{l}\text { Calidad del } \\
\text { trabaio. }\end{array}$ & Muy preciso & Diligente & Suficiente & Poco preciso & D eficiente & \\
\hline 5 & $\begin{array}{l}\text { Rapidez en } \\
\text { eiecución. }\end{array}$ & Muy rápido & Rápido & Normal & Lento & Muy lento & \\
\hline 6 & Iniciativa. & Mucha & Buena & Discreta & Escasa & Nula & \\
\hline 7 & $\begin{array}{l}\text { Espíritu de } \\
\text { colaboración. }\end{array}$ & Entusiasta & Muy dispuesto & D ispuesto & Indiferente & Retractario & \\
\hline 8 & Interés & $\begin{array}{l}\text { Mucho } \\
\text { interés }\end{array}$ & Preocupado & Normal & Poco interés & D espreocupado & \\
\hline 9 & $\begin{array}{l}\text { D ominio idiomas } \\
\text { extranieros }\end{array}$ & $\begin{array}{l}\text { Lee/ Escribe } \\
\text { / Habla }\end{array}$ & $\begin{array}{l}\text { Lee/ Escribe/ Ha } \\
\text { bla }\end{array}$ & Lee/ Escribe & Sólo Lee & \begin{tabular}{|l|} 
No \\
lee/ esc./ habla
\end{tabular} & \\
\hline 10 & $\begin{array}{l}\text { Act. Super. } \\
\text { Profesional. }\end{array}$ & Excelente & Buena & Suficiente & Poca & Ninguna & \\
\hline 11 & $\begin{array}{l}\text { D om. nuevas } \\
\text { tecnologías. }\end{array}$ & Total & Notable & Suficiente & Escaso & Nulo & \\
\hline & \begin{tabular}{|l|} 
Valoración \\
Global
\end{tabular} & 0 & 3 & 6 & 9 & 12 & \\
\hline 12 & $\begin{array}{l}\text { Comporta. en el } \\
\text { trabajo. }\end{array}$ & Intachable & Correcto & Disciplinado & Poca displ. & Indisciplinad. & \\
\hline 13 & Puntualidad & $\begin{array}{l}\text { Siempre } \\
\text { puntual }\end{array}$ & Evita retrasos & Retraso just. & Retr. Habitual & Siempre retras. & \\
\hline 14 & $\begin{array}{l}\text { Constancia en } \\
\text { trabajo. }\end{array}$ & $\begin{array}{l}\text { Excep. } \\
\text { Empeño } \\
\end{array}$ & Evita ausen. & Ausen. Just. & Aus. Habitual & Const. aus. & \\
\hline & \begin{tabular}{|l|} 
Valoración \\
Global
\end{tabular} & 0 & 2 & 4 & 6 & 8 & \\
\hline 15 & $\begin{array}{l}\text { Capacidad } \\
\text { orqanización }\end{array}$ & Excelente & Eficiente & Normal & Mediocre & Negativa & \\
\hline 16 & $\begin{array}{l}\text { Formación } \\
\text { subordinados }\end{array}$ & $\begin{array}{l}\text { Trab. } \\
\text { excelentes }\end{array}$ & Buenos trab. & Normal & No se ocupa & D esalienta & \\
\hline 17 & $\begin{array}{l}\text { Respeto de } \\
\text { subordinados }\end{array}$ & Excepcional & Bueno & Normal & Escaso & Inspira hostil. & \\
\hline 18 & $\begin{array}{l}\text { Asimilación } \\
\text { nuevos métodos }\end{array}$ & \begin{tabular}{|l|}
$\begin{array}{l}\text { Capta } \\
\text { rápidamente }\end{array}$ \\
\end{tabular} & \begin{tabular}{|l}
$\begin{array}{l}\text { Entiende } \\
\text { aclaraciones }\end{array}$ \\
\end{tabular} & $\begin{array}{l}\text { Necesita } \\
\text { tiempo adap. }\end{array}$ & $\begin{array}{l}\text { Entiende } \\
\text { parcialmente }\end{array}$ & Nula & \\
\hline & $\begin{array}{l}\text { Valoración } \\
\text { Global }\end{array}$ & $\mathbf{0}$ & 4 & 7 & 10 & 13 & \\
\hline & Valoración Total & $02-(0-2)$ & 3-11(3-7) & $12-20(8-12)$ & 21-20(13-16) & $29-32(17-20)$ & \\
\hline
\end{tabular}


Tabla 17

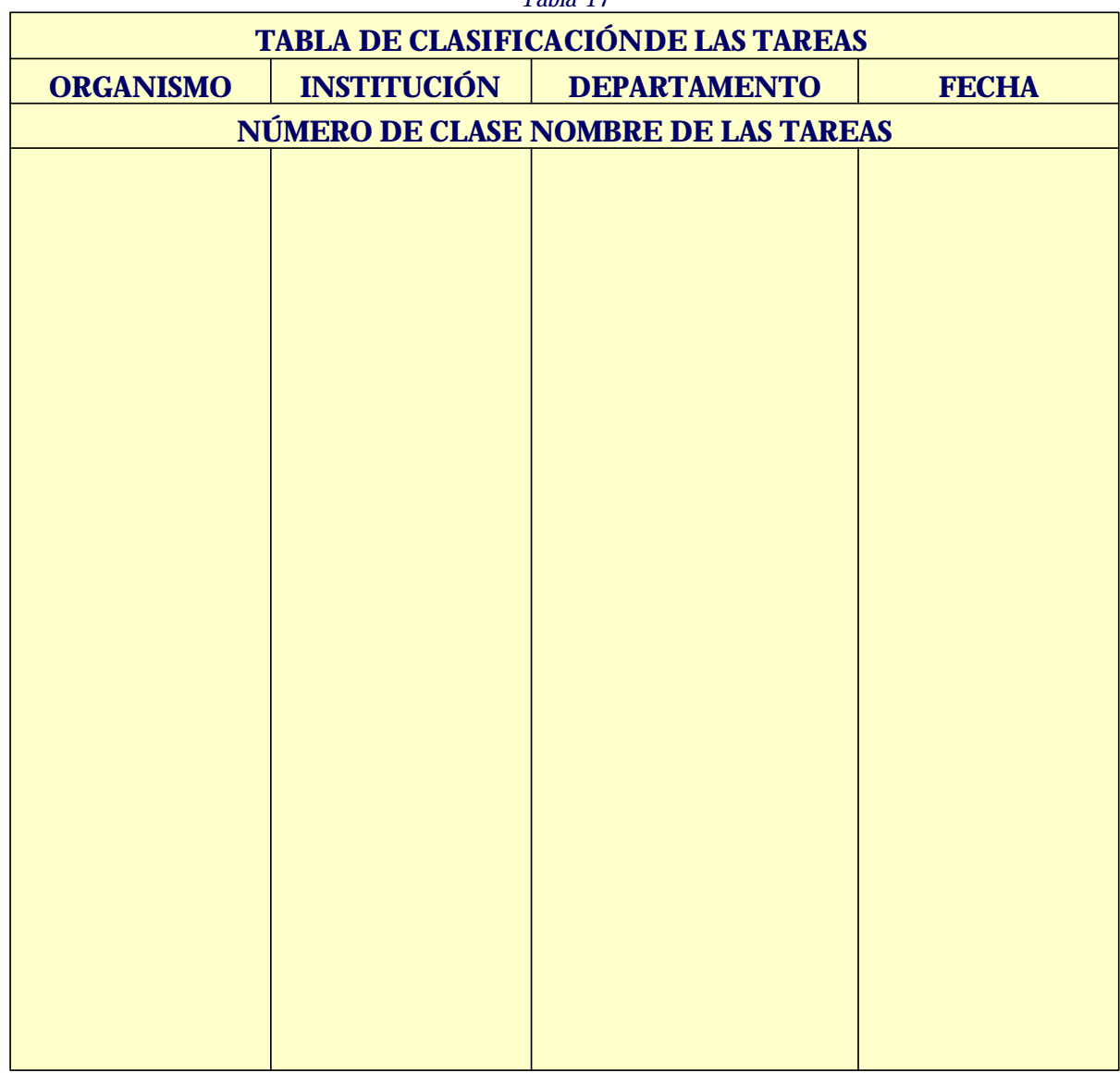

Tabla 18

Disposiciones a adoptar en el caso de presentarse abandono del puesto. (marcar con un asterisco)

\begin{tabular}{|c|c|c|c|c|}
\hline $\begin{array}{c}\text { Todo lo posible } \\
\text { porretenerlo }\end{array}$ & $\begin{array}{c}\text { Insistir en } \\
\text { retenerlo }\end{array}$ & $\begin{array}{c}\text { Nada en } \\
\text { particular }\end{array}$ & $\begin{array}{c}\text { Preferible dejar- } \\
\text { lomarchar }\end{array}$ & $\begin{array}{c}\text { Todo lo } \\
\text { posible por no } \\
\text { readmitinlo }\end{array}$ \\
\hline
\end{tabular}

FECHA

FIRMA DEL EVALUADOR

OBSERVACIO NES DE LA DIRECCION: 
T ablas auxiliares de puntuación

Tabla 19

\begin{tabular}{|c|c|}
\hline \multicolumn{2}{|c|}{ CAPACIDAD Y RENDIMIENTO DEL TRABAJO } \\
\hline PUNTUACIÓN & VALORACIÓN \\
\hline De 11 a 14 & Óntimo \\
\hline De 15 a 25 & Bueno \\
\hline De 26 a 36 & Medio \\
\hline De 37 a 47 & Escaso \\
\hline De 48 a 55 & Insuficiente \\
\hline
\end{tabular}

Tabla 20

\begin{tabular}{|c|c|}
\hline \multicolumn{2}{|c|}{ DISCIPLINA } \\
\hline PUNTUACIÓN & VALORACIÓN \\
\hline De3a4 4 & Óntimo \\
\hline De $5 \mathrm{a} 7$ & Bueno \\
\hline De $8 \mathrm{a} 10$ & Medio \\
\hline De $11 \mathrm{a} 13$ & Escaso \\
\hline De $14 \mathrm{a} 15$ & Insuficiente \\
\hline
\end{tabular}

Tabla 21

\begin{tabular}{|c|c|}
\hline \multicolumn{2}{|c|}{ ACTITUDES DIRECTIVAS } \\
\hline PUNTUACIÓN & VALORACIÓN \\
\hline De 4 a 6 & Óntimo \\
\hline De 7 a 11 & Bueno \\
\hline De12a 16 & Medio \\
\hline De17a 21 & Escaso \\
\hline De 22a 24 & Insuficiente \\
\hline
\end{tabular}

